\title{
Avirulence Gene Mapping in the Hessian Fly (Mayetiola destructor) Reveals a Protein Phosphatase 2C Effector Gene Family
}

Authors Names and Affiliations:

Chaoyang Zhao ${ }^{1,5}$

Richard Shukle ${ }^{2}$

zhao.1369@osu.edu

Lucio Navarro Escalante

shukle@purdue.edu

Mingshun $\mathrm{Chen}^{3}$

Stephen Richards ${ }^{4}$

navarroe@purdue.edu

Jeffrey J Stuart ${ }^{1}$

ming-shun.chen@ars.usda.gov

stephenr@bcm.edu

stuartij@purdue.edu

${ }^{1}$ Department of Entomology, Purdue University, West Lafayette, IN 47907

${ }^{2}$ USDA-ARS and Department of Entomology, Purdue University, West Lafayette, IN 47907

${ }^{3}$ USDA-ARS and Department of Entomology, Kansas State University, Manhattan, KS 66506

${ }^{4}$ Human Genome Sequencing Center, Baylor College of Medicine, Houston, TX 77030

Corresponding Author:

Jeffrey J Stuart

Department of Entomology, Purdue University, West Lafayette, IN 47907

stuartjj@purdue.edu

${ }^{5}$ Present Address: Department of Entomology, Ohio State University, Wooster, OH 44691 


\begin{abstract}
The genetic tractability of the Hessian fly (HF, Mayetiola destructor) provides an opportunity to investigate the mechanisms insects use to induce plant gall formation. Here we demonstrate that capacity using the newly sequenced HF genome by identifying the gene ( $v H 24)$ that elicits effector-triggered immunity in wheat (Triticum spp.) seedlings carrying HF resistance gene $\mathrm{H} 24$. $v H 24$ was mapped within a 230-kb genomic fragment near the telomere of HF chromosome X1. That fragment contains only 21 putative genes. The best candidate $v H 24$ gene in this region encodes a protein containing a secretion signal and a type- 2 serine/threonine protein phosphatase (PP2C) domain. This gene has an H24-virulence associated insertion in its promoter that appears to silence transcription of the gene in H24-virulent larvae. Candidate vH24 is a member of a small family of genes that encode secretion signals and PP2C domains. It belongs to the fraction of genes in the HF genome previously predicted to encode effector proteins. Because PP2C proteins are not normally secreted, our results suggest that these are PP2C effectors that HF larvae inject into wheat cells to redirect, or interfere, with wheat signal transduction pathways.
\end{abstract}

\title{
Graphical Abstract
}

First-instar Hessian fly larvae (A) use their microscopic mouthparts $(\mathrm{m})$ to introduce secreted serine/threonine protein phosphatases (B) into wheat cells. These effectors presumably modulate wheat signal transduction pathways that alter seedling growth and development. (C) Susceptible seedlings (S) are stunted, but seedlings that detect the presence of an effector induce effector-triggered immunity $(R)$ and grow normally.

\section{Key words}

\author{
Effector protein \\ Avirulence gene \\ Plant-gall \\ Gene-for-gene interaction \\ Plant resistance \\ Protein phosphatase
}




\section{Introduction}

Plant-galling arthropods, "nature's most sophisticated herbivores" (Shorthouse et al., 2005), create a protected nutrient-rich environment by modulating plant cell biochemistry and development. The resulting galls are often complex homeotic transformations of plant tissue. Occasionally they are so multifarious that they appear to be novel plant tissues. The precise mechanisms used to induce plant galls are poorly understood. However, evidence is accumulating that suggests that secreted effector proteins are responsible (Giron et al. this issue, Favery et al. this issue)(Aggarwal et al., 2014, Bent and Mackey, 2007, Harris et al., 2015, Hogenhout and Bos, 2011, Hogenhout et al., 2009, Stuart et al., 2012). This suggests that the same mechanisms that defend plants against certain pathogenic effectors issued by plant pathogenic microbes and nematodes are also used to defend plants against arthropod-delivered plant-galling effectors (Favery et al. this issue)(Harris et al., 2015, Hogenhout et al., 2009, Jones and Dangl, 2006, Kaloshian, 2004, Smith and Clement, 2012). Evidence supporting this hypothesis is accumulating (Aggarwal et al., 2014, Kobayashi et al., 2014, Zhao et al., 2015)(Kobayashi, this issue, Bentur et al., this issue).

One line of evidence that supports this hypothesis is the gene-for-gene interaction that has been observed between certain insects and their host plants (Stuart, 2015). This hypothesis has been extensively studied in plant pathology (Bent and Mackey, 2007), and the conceptual mechanisms underlying these relationships are familiar: Loosely analogous to antibody-antigen recognition in a vertebrate host, the products of specific plant resistance $(R)$ genes evolve to perceive, either directly or indirectly, specific plant pathogen effectors (Jones and Dangl, 2006). This perception elicits effector-triggered immunity (ETI) in the plant. The genes that encode these "perceivable" effectors are called Avirulence (Avr) genes because pathogen genotypes that express the effector are unable to colonize plants that carry the corresponding $R$ gene. The existence of $A v r$ genes in plant parasite populations is evidence that the parasite uses effectors to colonize its host. Avr gene mapping was the first method of effector discovery (Staskawicz et al., 1984), and it remains an effective approach (Ellis et al., 2009). Hundreds of effectorencoding genes are believed to reside in the genomes of plant-parasitic insects (Bos et al., 2010, Hogenhout and Bos, 2011, Zhao et al., 2015). A small number of these are Avr-encoded effectors.

$\mathrm{R}$ proteins may act as sentries that guard the cellular targets of parasite effectors or as decoys against effector activity (Jones and Dangl, 2006, van der Hoorn and Kamoun, 2008). They typically contain nucleotide binding (NB) and leucine rich repeat (LLR) motifs. A few proteins with these structures have been shown to protect plants from insects (Milligan et al., 1998, Rossi et al., 1998)(Kobayashi, this issue, Bentur et al., this issue). Normally the products of different $R$ genes perceive effectors that are encoded by different $A v r$ genes. Thus, a gene-forgene relationship is observed in which each plant $R$ gene has a different corresponding parasite Avr gene. When cognate $R$-Avr gene pairs are present in both host and parasite, ETI is elicited, resulting in a resistant, or incompatible, host-parasite interaction. In the absence of any other $R$ Avr gene pair, if either the $R$ or Avr cognate is missing, ETI is not induced. This results in a susceptible, or compatible, interaction. A very small number of plant-insect interactions exist in which such a gene-for-gene relationship has been demonstrated (Stuart, 2015) (Bentur et al. this issue, Kobayashi, this issue). However, it is also true that few plant-insect interactions have the genetic tractability necessary to detect these relationships.

One important plant-insect interaction that does have sufficient genetic tractability occurs between wheat (Triticum ssp.) and the gall-forming Hessian fly (HF, Mayetiola destructor). The $\mathrm{HF}$ is an important pest of a major commodity; so over 34 different HF $R$ genes have been 
genetically identified (Hao et al., 2013, Li et al., 2013, Li et al., 2015, McDonald et al., 2014). Methods to clone these genes in wheat are advancing (International Wheat Genome Sequencing, 2014). HF $R$ genes all have the designation " $H$ " (for HF) and then each is given a unique name or number, e.g. H13, H24 and Hdic. Genetic analyses performed on the HF suggest that for each $\mathrm{H}$ gene examined, a different cognate Avr gene exists in the insect (Stuart et al., 2012). These Avr genes are named by placing a "v" (for "virulence to") in front of the name of its cognate $R$ gene, e.g. $v H 13, v H 24$ and $v H d i c$, because recessive mutations in these Avr genes defeat resistance (Harris et al., 2003). Compatible and incompatible wheat-HF interactions can be observed on wheat seedlings, and individual seedlings can support multiple (>20) larvae. This makes it possible to establish and evaluate hundreds of wheat-HF interactions in a relatively small space. The gene-for-gene nature of the interaction was first observed using classical genetics. The ability to identify and clone a HF Avr gene was accomplished decades later, after the development of HF bacterial artificial chromosome (BAC) libraries and BAC-end sequencing (Aggarwal et al., 2014). The map-based approach used in that investigation has subsequently evolved with further advances in genetic technologies (Zhao et al., 2015). The approach also benefits from the insect's polytene chromosomes and its relatively small genome (Stuart et al., 2012). The HF reference sequence and associated genome browser (https://i5k.nal.usda.gov/Mayetiola destructor) facilitate this further, by making it possible to both easily develop molecular markers and identify genes in any region of the HF genome.

Although the HF-induced gall is not the characteristic outgrowth or swelling that is associated with many common galls, it is very much a gall in the sense that the physiology of the plant is altered and a nutrient tissue is created (Harris et al., 2010, Harris et al., 2006, Harris et al., 2003). The plant acts as the source of photosynthates and other nutrients and the developing HF larvae act as the sink that receives those substances (Harris et al., 2015, Stuart et al., 2012). In only 4 days, a single larva ( $<1 \mathrm{~mm}$ in length), secretes powerful substances that permanently cause the entire seedling to cease cell elongation, stop cell division, dramatically alter plant metabolism and severely increase cell permeability; all for the benefit of the larva (Liu et al., 2007, Stuart et al., 2012, Williams et al., 2011, Zhu et al., 2008). Increasing the expression of at least one wheat gene, Md susceptibility-1 (Mds-1), is critical to larval survival and plant stunting (Liu et al., 2013). We suspect, therefore, that altering the expression of Mds-1 and other "susceptibility genes" may be the mechanism HF effectors use to modulate plant developmental pathways and that this is key to the power of the larval secretions.

To explore the hypothesis that insect effectors are used to induce plant-gall formation, candidate effectors have been identified in the larval salivary gland transcriptome (Chen et al., 2010) and the HF genome has been sequenced (Zhao et al., 2015). We have also exploited the genetic tractability of the HF to genetically map effector-encoding Avr genes (Aggarwal et al., 2014, Zhao et al., 2015). Our intent here is to describe the facility of map-based Avr gene discovery in the HF as a means of identifying insect proteins that are capable of reprograming plant development and immunity. These methodologies will be reviewed as a novel candidate HF Avr gene, $v H 24$, is mapped and described. $v H 24$ encodes the effector that elicits ETI in wheat seedlings carrying $R$ gene $H 24$. It is presumed that this effector benefits the insect as a virulence factor in the absence of $\mathrm{H} 24$. $\mathrm{H} 24$ itself was identified in the diploid wheat $T$. tauschii, and transferred into bread wheat (T. aestivum) via a wide cross (Raupp et al., 1993). It was mapped with respect to molecular markers on the long arm of wheat chromosome 3D (Ma et al., 1993). Although it has not been deployed in wheat cultivars in the United States, surveys of U.S. HF populations indicate that $\mathrm{H} 24$-virulence is not uncommon (Chen et al., 2009). 
The ability to perform map-based Avr gene identification in the HF puts insect plant parasites on par with microbial plant pathogens, whose smaller genomes and rapid reproduction have permitted Avr gene cloning for decades (Staskawicz et al., 1984). Although these are not the only methods that can be applied to identify proteins and other molecules that plant parasites use to modulate plant development and immunity, they remain an effective and tested means of effector identification. Modern sequencing technologies are rapidly adding sequenced insect genomes to the arsenal of tools we have to investigate plant-insect interactions (Richards and Murali, 2015). By combining these technologies with other plant-parasitic insects that are amenable to genetics, we can expect the approach to become more effective in the identification of genetic traits that underlie the phenotypic differences that are observed among many plant-insect interactions. Our understanding of gene-for-gene and other plant-insect interactions will certainly benefit, as the contributions of Kobayashi () and Bentur et al. () in this issue clearly attest.

\section{Materials and Methods}

\subsection{H24-virulent, H24-avirulent and structured HF mapping populations}

All HFs were reared as the offspring of individual females on separate caged pots of wheat seedlings as previously described (Rider et al., 2002). All HF strains, matings and families were reared at $18{ }^{\circ} \mathrm{C}$ under a 12 -h light: 12 -h dark photoperiod. H24-virulent and H24-avirulent strains were selected from an Israeli HF strain maintained in the USDA HF research program at Purdue University.

To select the H24-avirulent strain, single mated females from the Israeli HF population were allowed to deposit their eggs on leaves of wheat seedlings growing in 36 caged pots. Each pot contained HF-susceptible 'Newton' seedlings growing on one side of the pot and the H24resistant seedlings in the other side of the pot. The seedlings and the larvae in each pot were examined 10 days after egg deposition for plant stunting and larval survival. Pots that had either stunted H24-seedlings or surviving larvae on H24-seedlings were discarded. Larvae on the Newton seedlings on the remaining pots were allowed to develop, emerge as adults and intermate. Individual mated females were then caged on separate pots of Newton and H24seedlings for a second generation of selection for H24-avirulence. This procedure was followed for three generations, after which there was no evidence of $H 24$-virulence in the population. This population was subsequently maintained as the "H24-avirulent strain" reared as offspring of single females on caged pots of Newton seedlings.

To select the H24-virulent strain, we followed standard procedures (Zantoko and Shukle, 1997). Briefly, mated HFs collected from the Israeli population deposited their eggs on H24-resistant seedlings. Surviving larvae on stunted seedlings were allowed to develop into adults. These adults were allowed to intermate and the mated females deposited their eggs on H24-resistant seedlings in caged pots. This process was repeated for a third generation in order to increase the frequency of $\mathrm{H} 24$-virulence. The first-instar larval offspring of the surviving females were then collected individually in $10 \mu \mathrm{L}$ drops of water containing $0.02 \% \mathrm{NP}-40$ and placed, one larva per plant, on the youngest leaf of single H24-seedlings. Using a single larva per plant insures that avirulent larvae, which can survive on the nutritive tissue created by a virulent larva on the same plant (Harris et al., 2006, Williams et al., 2011), are eliminated from the population. Larvae that survived alone on the $\mathrm{H} 24$ seedlings were used to establish the H24-virulent strain. This strain was maintained on H24-resistant plants under the conditions described above.

Three independent structured mapping populations (F2A, F2B and F4C) were developed from crosses between virulent and avirulent males and females as previously described (Aggarwal et 
al., 2014). HF females typically produce offspring of only one sex (Benatti et al., 2010). Therefore each mapping population was initiated by crossing two $\mathrm{P}_{1}$ sister H24-virulent $(v / v)$ females to the same $\mathrm{P}_{1} \mathrm{H} 24$-avirulent $(A /-)$ male (Figure $\left.1 \mathrm{~A}\right)$. One $\mathrm{P}_{1}$ female produced $\mathrm{F}_{1}$ daughters and the other produced $F_{1}$ sons. After mating and egg deposition, the $P_{1}$ male and females were collected and the genomic DNA of each $P_{1}$ HF was extracted separately. The $F_{1}$, and all subsequent generations, were reared on Newton seedlings and allowed to freely intermate. To map H24-virulence relative to molecular genetic markers, males were collected from the $\mathrm{F}_{2}$ and $\mathrm{F}_{4}$ generations and individually genotyped as H24-virulent or H24-avirulent in separate testcrosses (Figure 1B). Males were collected immediately after mating and placed at $80{ }^{\circ} \mathrm{C}$ in separate $0.5 \mathrm{~mL}$ plastic tubes for future genomic DNA extraction.

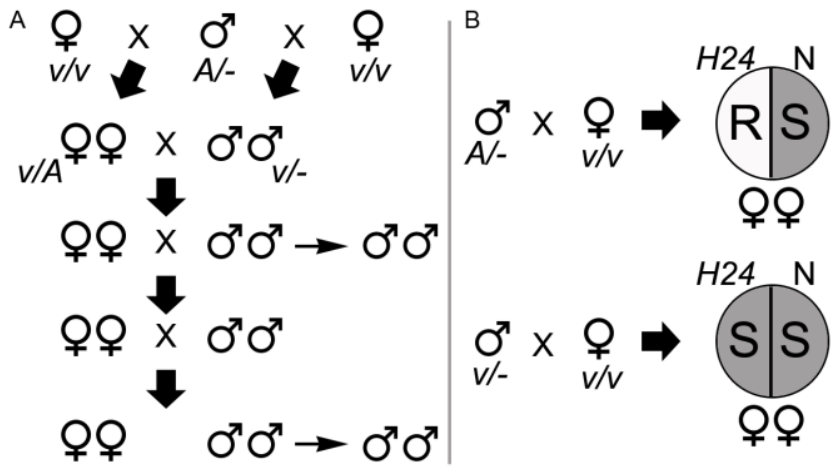

Fig. 1. HF males taken from structured mapping populations are genotyped as virulent or avirulent to HF $R$ gene $H 24$ in wheat. (A) Mapping populations are initiated with matings between homozygous virulent $(v / v)$ sister females and a single avirulent $(A /-)$ male. Their offspring are allowed to freely intermate. Males are collected for genotyping after the $\mathrm{F}_{1}$ generation (horizontal arrows). (B) Collected males are individually genotyped in testcrosses with homozygous virulent females $(\mathrm{V} / \mathrm{V})$. H24-avirulent $(A /-)$ males produce female offspring that are incapable of stunting $H 24$-plants (R). H24-virulent ( $\mathrm{V} /$-) males produce female offspring that are capable of stunting H24-resistant plants (S). Newton (N) wheat seedlings are used as a susceptible check.

Testcrosses consisted of individual males mated to a single homozygous $H 24$-virulent females, collected from the H24-virulent strain, caged together on separate pots containing $\mathrm{H} 24$ and Newton seedlings growing in separate halves of the pots (Figure 1B). The Newton seedlings served as a check for infestation. Because each female produced offspring of only one sex, the adults that emerged from the Newton seedlings allowed us to determine the sex of each testcross family. If the testcross offspring were female and unable to live on $\mathrm{H} 24$ plants, the male was scored as "H24-avirulent." If the testcross offspring were female and able to live on $\mathrm{H} 24$ plants, the male was scored as "H24-virulent." If the testcross offspring were males, the genotype of the male could not be determined.

\subsection{Molecular genetic mapping}

Molecular markers were developed and genetic and physical mapping were performed as described previously (Aggarwal et al., 2009, Aggarwal et al., 2014). Briefly, primers flanking simple sequence repeats (SSRs) in genomic sequence were designed and synthesized to produce short amplicons (100 to $400 \mathrm{bp}$ ). These were used to PCR-amplify genomic DNA isolated from males genotyped as $\mathrm{H} 24$-virulent and $\mathrm{H} 24$-avirulent in separate reactions. We found that $28 \%$ of the primer pairs flanking SSRs on the $X$ chromosomes produced amplicons with polymorphic lengths visible on $2.5 \%$ agarose gels (Table S1). Primers used to position H24-virulence within scaffold X1R.8 are presented in Table S2. The amplicons produced using 
$\mathrm{P}_{1}$ male and female genomic DNA determined the phase of the polymorphisms. HF bacterial artificial chromosomes (BACs) containing the polymorphic sequences were used as probes against the HF polytene chromosomes in fluorescence in situ hybridization (FISH) experiments in order to confirm the genomic locations of the markers. Sequenced BAC-ends allowed us to determine the location of the BACs in HF sequenced scaffolds.

\subsection{Analysis of candidate $\mathrm{vH} 24$ gene transcription}

Total RNA was isolated from two-day-old first-instar larvae of the H24-avirulent strain using the RNeasy Mini Kit (Qiagen). The sequences of the oligonucleotide primers used in RT-PCR experiments are presented in Table S3. To synthesize the first-strand cDNA, $0.5 \square \mathrm{g}$ of total RNA was mixed with 10 pmol of SMART IV oligo DNA and 10 pmol of (dT)17-adaptor oligo DNA (Table S3) in a total volume of $5 \square \mathrm{l}$. The mixture was heated to $72^{\circ} \mathrm{C}$ for $2 \mathrm{~min}$. and then chilled on ice for $2 \mathrm{~min}$. To synthesize the full-length first-strand cDNA, we used the SMARTScribe kit (Clonetech) according to the manufacturer's instructions. Briefly, $5 \mu \mathrm{L}$ of first-strand buffer, $1 \mu \mathrm{L}$ of $20 \mathrm{mM} \mathrm{DTT}, 1 \mu \mathrm{L}$ of dNTP $\operatorname{mix}(10 \mathrm{mM})$, and $1 \mu \mathrm{L}$ of SMARTScribe MMLV reverse transcriptase (Clontech) were added to the tube to bring the total volume to $10 \mu \mathrm{L}$. The mixtures were incubated at $42^{\circ} \mathrm{C}$ for $1 \mathrm{~h}$ using the SMART IV oligo to amplify from the 5 '-end and the (dT)17-adaptor to amplify from the 3 '-end of the single stranded transcripts. To generate fulllength double-stranded cDNA, long-distance PCR was performed using the Advantage 2 polymerase Mixes (Clontech), as recommended by the manufacture, and forward primer 5'RACE-F and reverse primer (dT)17-adaptor. PCR conditions were $95^{\circ} \mathrm{C}$ for $1 \mathrm{~min}$ followed by 22 cycles of $95^{\circ} \mathrm{C}$ for $15 \mathrm{~s}$ and $68^{\circ} \mathrm{C}$ for 6 min.

Rapid Amplification of cDNA Ends (RACE) was performed in both the $5^{\prime}$ and $3^{\prime}$ directions as nested PCR experiments. The initial 5'-RACE was performed using the forward primer 5'RACE-F, the reverse primer Mdes001440-5RACE-R1 and synthesized double-stranded cDNA. The nested PCR used primers 5'-RACE-F and Mdes001440-5RACE-R2 and the product from the initial $5^{\prime}$ reaction as template. To perform 3'-RACE, the first reaction used primers Mdes001440-3RACE-F1 and 3'-adaptor-R. The nested reaction used primers Mdes001440-F and $3^{\prime}$-adaptor- $R$ and product from the initial $3^{\prime}$-reaction as template. The amplicons produced were cloned into plasmid vector pCR4-TOPO (Invitrogen) and sequenced at the Purdue Genomics Center.

\subsection{PCR-walking}

To clone and sequence genomic DNA upstream of the virulence-associated allele of candidate gene Mdes001440, we used TOPO-vector-ligation PCR (TVL-PCR) to perform a short sequence walk (Orcheski and Davis, 2010). To prepare template for TVL-PCR, $0.6 \mu \mathrm{g}$ of H24virulent genomic DNA was digested to completion with EcoRI (New England Biolabs) and then end-repaired using GoTaq DNA polymerase (Promega) according to the manufacturer's instructions. This created 3'-adenine-overhangs on each DNA fragment. The fragments were cleaned using the QIAquick PCR purification kit (Qiagen) and ligated into the pCR4-TOPO vector (Invitrogen). Nested PCR was then performed using this template. Two separate firstround reactions were performed, using oligonucleotides complementary to the M13F and M13R sites in separate reactions with the candidate Mdes001440-specific oligonucleotide Mdes001440-R. The products of these reactions were used as template in two separate PCR experiments. The first used an oligonucleotide complementary to the T7 site and primer Mdes001440-R1. The second used an oligonucleotide complementary to the T3 primer site and Mdes001440-R1. The amplicons generated in these experiments were cloned into plasmid vector pCR4-TOPO and sequenced at the Purdue Genomics Center. 


\section{Results}

\subsection{An Israeli HF population permits the isolation of $\mathrm{H} 24$-virulent and $\mathrm{H} 24$-avirulent strains}

Over several decades, HF strains, races or "biotypes" have been selected from natural populations that differ with respect to their ability to survive on and stunt wheat seedlings with different genes for resistance (Gallun et al., 1961). These have been used to both identify new $R$ genes and to determine the effectiveness of $R$ genes bred into elite cultivars. Virulence to H24 was extremely low to non-existent in the Purdue University greenhouse populations. We therefore sought $\mathrm{H} 24$-virulence in a population collected in Israel and maintained in the USDAARS greenhouse at Purdue University. We reasoned that chromosome rearrangements might interfere with recombination analyses involving crosses between U.S. and Israeli HF populations. To avoid this possibility, a complementary H24-avirulent population (AvrH24) was isolated from the same Israeli HF population.

Approximately $1000 \mathrm{H} 24$-seedlings were infested with 150 females arbitrarily selected from the Israeli population. The females deposited all of their eggs (>2000) on the H24-seedlings and the eggs were allowed to eclose and the neonate larvae were allowed to attempt to establish nutritive tissue at the base of the plants. Among all of the infested plants, 15 had a susceptible reaction. Assuming the susceptibility of each of these plants was due to a single H24-virulent larva, we estimated that less than $1 \%$ of the Israeli population is $H 24$-virulent. Larvae surviving on the susceptible seedlings were allowed to develop into adults and used to create an $\mathrm{H} 24$ virulent strain (virH24) as described in the Materials and Methods. We detected no phenotypic differences in compatible interactions between H24-virulent and H24-avirulent larvae on Newton seedlings.

\subsection{H24-virulence is a recessive $X$-linked trait}

Understanding the unusual inheritance pattern in the HF is essential to mapping virulence mutations (Fig. 2). By convention, the chromosomes composing a HF zygote's genome are grouped into eight "S" and a variable number (28-32) of "E" chromosomes (Stuart et al., 2012). The S chromosomes are divided further into two autosome pairs (A1 and A2) and two " $X$ " chromosome pairs (X1 and X2). S chromosomes undergo meiotic recombination in females, are sexually inherited and are present in both the soma and the germ line. The $E$ chromosomes are maternally inherited and germ-line-limited. Because they have no impact on the inheritance of virulence, they are given little attention here.

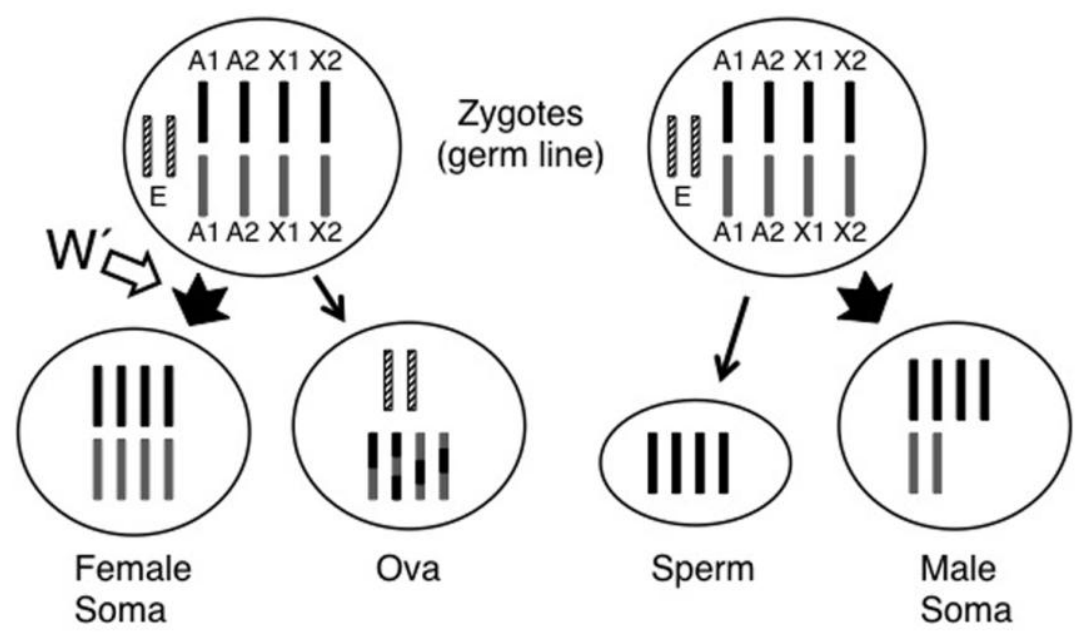


Fig. 2. Chromosome elimination and sex determination in the Hessian fly. S chromosomes ( $A 1, A 2, X 1$ and $X 2)$ are shown as black (maternally inherited) and grey (paternally inherited) vertical lines. E chromosomes are shown as vertical hatched lines.

Zygotes receive one copy of each $S$ chromosome from their father (A1A2X1X2). Because males transmit only their mother's S chromosomes, all of the offspring of a single male are isogenic with respect to these chromosomes. Zygotes receive one copy of each S chromosome and a complement of $E$ chromosomes from their mother (A1A2X1X2E, where $E$ represents 28-32 E chromosomes). Because S-chromosome recombination occurs during oogeneisis, zygotes differ with respect to alleles on these chromosomes. Chromosome elimination during embryogenesis determines the sex of an individual; individuals that retain a diploid complement of the $S$ chromosomes (A1A2X1X2/A1A2X1X2) develop as females, but individuals that eliminate the paternally derived $X$ chromosomes (A1A2X1X2/A1A2OO) develop as males. Maternal genotype determines whether the paternal $X$ chromosomes are retained or eliminated (Benatti et al., 2010). Females carrying a small autosomal inversion ( $\left.W^{\prime}\right)$ cause paternal $X$-chromosome retention (Fig. 2). As a consequence, all of the offspring of $W^{\prime}$-bearing females develop as females. Females lacking $W^{\prime}$ condition their offspring to eliminate the paternally inherited $X$ chromosomes, and as a result, all of their offspring develop as males.

Utilizing this knowledge, virulence can be readily assigned to autosomes or $\mathrm{X}$ chromosomes as a recessive trait (Fig. 3). If matings between virulent females and avirulent males produce avirulent male offspring, virulence is autosomal. However, if those matings produce virulent male offspring, virulence is $\mathrm{X}$ linked.

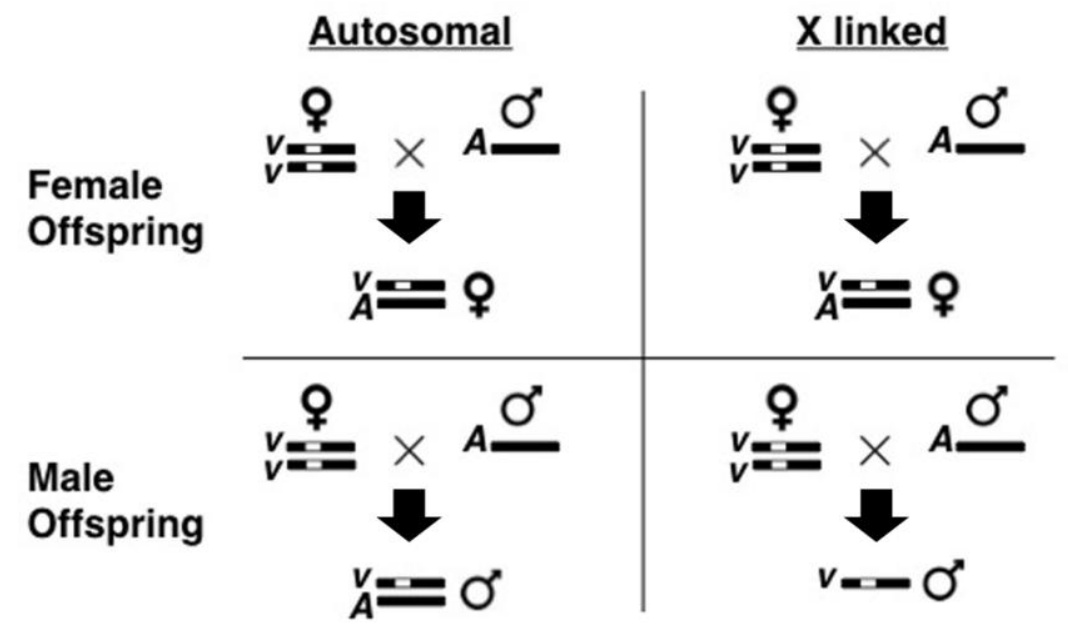

Fig. 3. All possible male and female genotypes resulting from crosses between homozygous virulent $(v / v)$ females and avirulent $(A /-)$ males are illustrated. When the virulence mutation is $X$ linked, male offspring are able to survive on resistant plants because they lack the dominant avirulent allele $(A)$.

We made 13 independent crosses between AvrH24 males and virH24 females. Each mated female was allowed to oviposit on a caged pot containing H24 and 'Newton' (fully susceptible) seedlings on opposite sides of the pot. Newton seedlings were provided to assure that some $F_{1}$ offspring in each $F_{1}$ family survived to the adult stage so that the sex of each family could be easily determined. A family was considered $\mathrm{H} 24$-virulent if the larvae survived on and stunted $\mathrm{H} 24$ seedlings. A family was considered $\mathrm{H} 24$-avirulent if the larvae died and failed to stunt $\mathrm{H}_{24}$ seedlings. We observed only two types of $F_{1}$ families: seven were $H 24$-avirulent and female, and six were $\mathrm{H} 24$-virulent and male. These data indicate that $\mathrm{H} 24$-virulence is an X-linked 
recessive trait, and are consistent with the hypothesis that $H 24$-virulence is conditioned by lossof-function mutations in a single, X-linked, effector-encoding Avr gene, vH24.

\subsection{H24-virulence resides within a 230-kb sequence near an X1 telomere}

To determine the chromosomal position of H24-virulence, we used PCR-based molecular markers and DNA isolated from $F_{2}$ males collected from mapping population $F 2 A(n=38)$ and genotyped in separate testcrosses with homozygous $H 24$-virulent females (Fig. 1B). We began by performing bulked segregant analysis at 46 markers distributed on chromosomes X1 and X2 (Table S1). An H24-virulent DNA pool was prepared by mixing 40 ng of DNA extracted from each of 10 virulent $F_{2}$ males collected from the F2A population. An H24-avirulent DNA pool was prepared in a similar manner. We then compared the amplicons produced using these pools as DNA template for each marker. A quantitatively uneven distribution of polymorphic bands was suggestive of a marker linked to H24-virulence (Fig. 4A). We observed such an indication with respect to only three markers, each located on scaffold X1.7 (markers X1.7-95, X1.7-166 and X1.7-305), which had been previously positioned near the telomere of chromosome X1 (Aggarwal et al., 2009). To verify that these markers were linked to H24-virulence, we subsequently examined all 38 genotyped $F_{2}$ males in the F2A population separately with respect to the segregation of the polymorphisms associated with these markers (Fig. 4B). This analysis indicated that $\mathrm{H} 24$-virulence was most closely linked to the most distal X1.7 marker $(\mathrm{X} 1.7-95,+6)$ and suggested that $H 24$-virulence mutations reside on a more distal X1 scaffold.

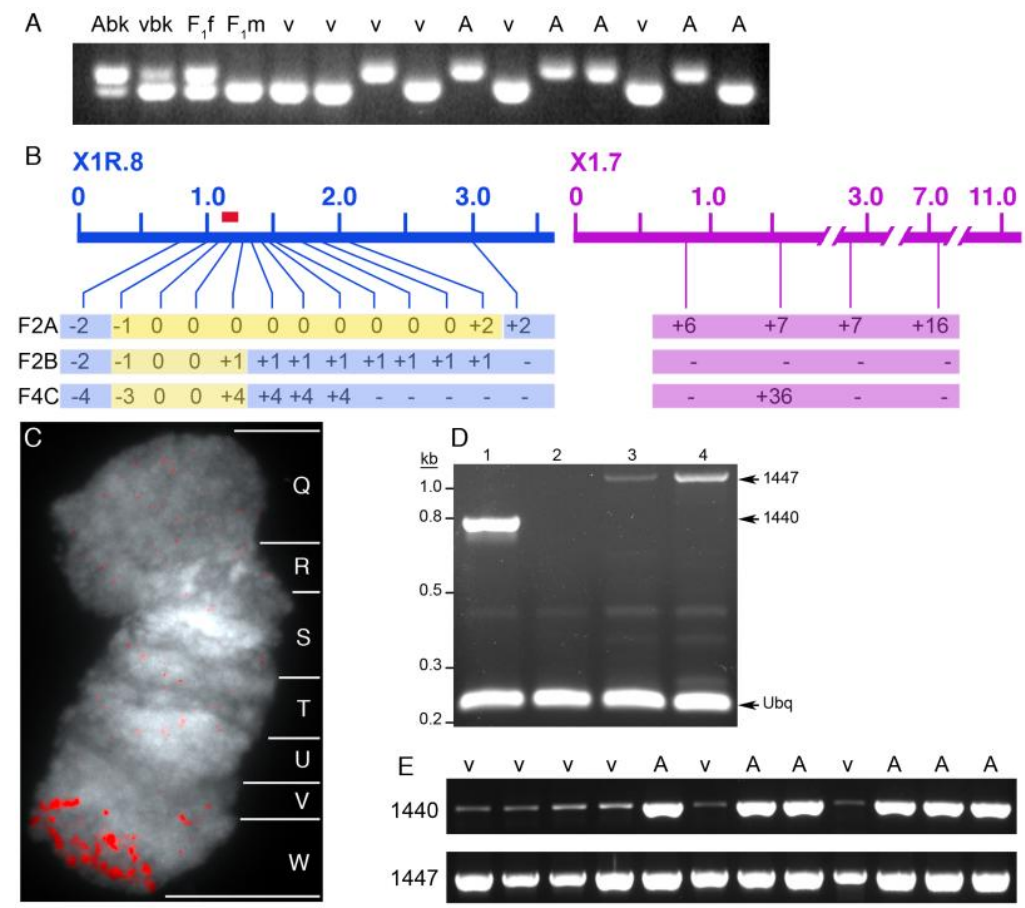

Fig. 4. Mapping $v H 24$ in the HF genome. (A) Example of a scaffold X1.7 marker amplified in H24-avirulent (Abk) and -virulent (vbk) bulks, an F1 female (F1f), an F1 male (F1m) and a F2 virulent (v) and avirulent (A) few individuals. (B) Scaffold maps showing the number of recombinant individuals in mapping populations F2A, F2B and F4C markers in scaffolds X1R.8 and X1.7 (upper scale is Mb). With respect to the chromosome, markers to the left are more distal (further from the centromere) than markers on the right. (C) Polytene chromosome X1 showing the divisions based on chromosome banding and the red fluorescence associated with the hybridization of an X1R.8 BAC (red bar in B). (D) RT-PCR amplification of genes Mdes001440 and Mdes001447 using RNA extracted from H24-avirulent (lanes 1 and 3 ) and H24-virulent (lanes 2 and 4) first-instar larvae. Primers used to amplify HF Ubiquitin (Ubq) were used as 
internal controls in each reaction. (E) PCR amplification of Mdes001440 and Mdes001147 using DNA extracted from H24-virulent and -avirulent individuals. Note that the virulence-associated DNA fails to amplify well with primers designed to amplify Mdes001440, but there is no such association with Mdes001447.

To test this possibility, we considered each of six scaffolds that had previously been physically positioned in the "W" segment of chromosome X1 (Fig. 4C), but had not been ordered with respect to other scaffolds on the chromosome (Aggarwal et al., 2009). Scaffold X1Random.8, the longest of the six, was examined first. Fluorescence in situ hybridization of X1Random.8 $\mathrm{BAC}$ clones indicated the scaffold was very near the $\mathrm{X} 1$ telomere (Fig. $4 \mathrm{C}$ ). Using the F2A population, the genetic positions of thirteen X1Random.8 markers (Table S2) were determined. These data confirmed that X1Random. 8 is distal to scaffold X1.7 (Fig. 4B). In addition, nine markers within a 620-kb X1Random. 8 segment failed to recombine with H24-virulence, suggesting that mutations conditioning H24-virulence reside between markers X1R.8-81 and X1.R.8-148 on the X1Random.8 scaffold.

To test this possibility further, we developed two additional mapping populations, F2B and F4C. The F2B population consisted of $39 \mathrm{~F}_{2}$ males and the $\mathrm{F} 4 \mathrm{C}$ population consisted of $66 \mathrm{~F}_{4}$ males. Each male was genotyped with respect to $\mathrm{H} 24$-virulence and polymorphisms at scaffold X1.7 and X1Random.8 markers (Fig. 4B). These populations resolved the position of $\mathrm{H} 24$-virulence to a 230-kb sequence between markers X1R.8-81 (base 1,121,087) and X1R.8-99 (base $1,351,496)$, within the $620-k b$ sequence identified using population F2A (Table S2). This result was consistent with the hypothesis that loss-of-function mutations in a single effector-encoding Avr gene defeat $\mathrm{H} 24$-resistance.

\subsection{Genic analyses reveal a single vH24 candidate}

To test this hypothesis further, we identified the genes between markers X1R.8-81 and X1R.899 and examined them for a signal peptide, a feature that is commonly associated with eukaryotic effectors. Importantly, the interplay $R$ and $A v r$ genes during the coevolution of host and parasite can result in diversifying selection for alleles at both loci (Allen et al., 2004). We therefore used evidence of diversifying selection, an absence of sequence similarities to other proteins, to help us identify candidate HF Avr genes.

Using Web Apollo (Lee et al., 2013) and the HF sequence data maintained at the i5k workspace (https://i5k.nal.usda.gov/Mayetiola destructor), 21 putative genes were discovered and manually annotated within the H24-virulence-associated 230-kb segment (Table 1). The signal peptide prediction algorithm, SignalP4.1 (Petersen et al., 2011), indicated that only four of these genes encode signal peptides. BLASTP (Altschul et al., 1990) sequence alignments indicated that only one of these genes (Mdes001440) has the low sequence conservation expected of a rapidly evolving effector-encoding Avr gene. Mdes001440 is composed of a single exon, and this encodes a protein that has less than $27 \%$ amino acid identity with its best hit, a vertebrate type $2 \mathrm{C}$ protein phosphatase (PP2C; also known as metal ion-dependent protein phosphatases, PPM). It lacks significant amino acid sequence similarities to all arthropod genes in the NCBI non-redundant protein database.

Expression analysis supported the hypothesis that Mdes001440 is $\mathrm{vH} 24$. Using total RNA separately extracted from $\mathrm{H} 24$-virulent and $\mathrm{H} 24$-avirulent larvae, reverse-transcription PCR, (RT-PCR) was performed using primers designed to specifically amplify transcripts of Mdes001440 (primers: Mdes001440-F and Mdes001440-R; Table S3) and Mdes001447 (primers: Mdes001447-F and Mdes001447-R). Consistent with predicted Avr gene loss-offunction, there was no evidence of Mdes001440 transcription in H24-virulent first-instar larvae, although transcript amplification was evident in H24-avirulent first-instar larvae (Fig. 4D). 
Mdes001447 expression was evident in both H24-virulent and H24-avirulent larvae, as was the expression of the internal control (HF ubiquitin) in all reactions.

Table 1

Genes residing within the 230-kb H24-virulence-associated region of the HF genome.

\begin{tabular}{lllllll}
\hline Mdes no. & Position $^{2}$ & Length $^{3}$ & SP $^{4}$ & BLASTp hit & Locus & E-value \\
\hline 001435 & +1167578 & 1323 & + & Ccap; dystroglycan-like protein DG & XP_004535783 & 0.0 \\
001436 & +1183611 & 743 & - & Dgrim; GJ20080 & XP_001987700 & $6 \mathrm{e}-103$ \\
001437 & -1185696 & 174 & - & Mdom; ATP synthase subunit d & XP_005175277 & $4 \mathrm{e}-62$ \\
001438 & +1189873 & 174 & - & Amel; ras-like GTP-binding protein & XP_006570782 & $7 \mathrm{e}-76$ \\
001439 & +1183271 & 440 & - & None & None & None \\
001440 & -1203662 & 266 & + & Trub; serine/threonine phosphatase & XM_003975587.1 & 0.004 \\
001441 & +1206881 & 891 & - & Ccap; transportin-1-like & XP_004526941 & 0.0 \\
001443 & -1219619 & 239 & - & Cquin; transport protein Use1 & XP_001841767 & $4 \mathrm{e}-81$ \\
001444 & +1219983 & 597 & - & Dmel; separase & NP_523935 & $1 \mathrm{e}-40$ \\
001445 & -1228195 & 1272 & - & Cflor; TE P transposase & EFN62483 & $5 \mathrm{e}-22$ \\
001446 & +1229135 & 214 & - & Dmel; Rab19-like protein & NP_523970 & $9 \mathrm{e}-85$ \\
001447 & +1237602 & 369 & + & Dmel; CG7201, isoform A & NP_648200 & $7 \mathrm{e}-100$ \\
001448 & +1276852 & 881 & - & Aaeg; neurotactin & XP_001659857 & 0.0 \\
001449 & -1293243 & 1930 & + & Dmel; plexin A, isoform A & NP_524637 & 0.0 \\
001450 & -1304015 & 345 & - & Dmel; UDP-sugar transporter & BAB62105 & $3 \mathrm{e}-156$ \\
001451 & +1298401 & 302 & - & Cquin; NTKL-binding protein 1 & XP_001870841 & $6 \mathrm{e}-45$ \\
001452 & -1301589 & 261 & - & Aaeg; ubiquinone biosynthesis protein & XP_001649247 & $1 \mathrm{e}-116$ \\
001453 & -1308530 & 369 & - & Aaeg; spermine synthase & XP_001653177 & $9 \mathrm{e}-102$ \\
001454 & +1311513 & 725 & - & Aaeg; type iv inositol 5-phosphatase & XP_001653179 & 0.0 \\
001455 & +1317832 & 874 & - & Mdom; palmitoyltransferase-like & NP_001137938 & 0.0 \\
001457 & +1329733 & 411 & - & Cquin; lim homeobox protein & XP_001843929 & $1 \mathrm{e}-124$ \\
\hline
\end{tabular}

${ }^{1}$ After manual annotation of the Mdes 1.0 official gene set.

${ }^{2}$ Base corresponding to translation start site in scaffold X2Random.8; positive (+) and negative (-) strands.

${ }^{3}$ Number of amino acid residues.

${ }^{4}$ Signal peptide present (+) or absent (-)

${ }^{5}$ Aaeg $=$ Aedes aegypti, Amel $=$ Apis mellifera; Ccap = Ceratitis capitata, Cflor = Camponotus floridanus, Cquin = Culex quinquefasciatus, Dgrim = Drosophila. grimshaw; Dmel = D melanogaster, Mdom = Musca domestica; Trub= Takifugu rubripes (tiger puffer fish)

We also performed 5'- and 3'- RACE to obtain the complete sequence of Mde001440 transcripts. The result was in agreement with our previous genomic annotation of the gene (Fig. S1). The Mdes001440 transcript is 1026 bp long and contains an 801-bp coding region. Its predicted protein is composed of 266 amino acid residues, which includes a putative secretory signal of 25 amino acids at the N-terminus (Fig. S1). Although Mdes001440 has little sequence similarity to genes in other organisms, structural alignments indicated that it encodes a protein with an imperfect PP2C Serine (Ser)/Threonine (Thr) protein phosphatase domain (Fig. 5). In this alignment, only one of the eight residues present at the active site failed to map to the residues that compose the canonical PP2C domain.

Oligonucleotide primers used to analyze Mdes001440 and Mdes001447 gene expression were used to amplify genomic copies of the genes from both $\mathrm{H} 24$-virulent and $\mathrm{H} 24$-avirulent individuals. These experiments indicated that allelic sequence variation exists between $\mathrm{H} 24$ virulent and H24-avirulent individuals at the Mdes001440 locus (Fig. 4E). To discover the source of this variation, we cloned Mde001440 from DNA isolated from H24-virulent individuals using a combination of PCR and TVL-PCR sequence walking. A 163-bp DNA insertion, 134-bp upstream of the transcription start site (Fig. S1), was the only difference observed between virulent- and avirulent-associated sequences. This insertion is flanked by an 8-bp short direct repeat followed by a 4-bp inverted repeat and is present in seven different scaffolds of the HF reference sequence (BLASTN $\leq 4 \mathrm{e}-81$ ), suggesting that it is the remnant of a class II 
transposable element. The insertion presumably changes the tertiary conformation of the gene, which interferes with both its transcription (Fig. 4D) and PCR amplification (Fig. 4E).
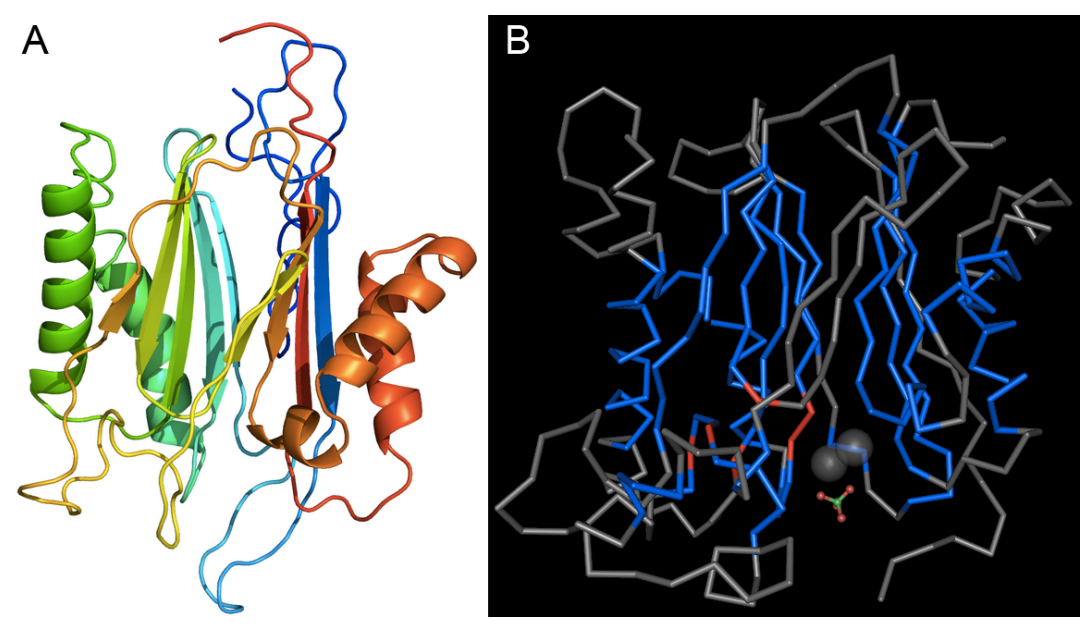

Fig. 5. Predicted structure of the protein encoded by candidate $v H 24$ gene Mdes001440. (A) Phyre2 (Kelley and Sternberg, 2009) predicted structure of 191 out of 266 amino acids modeled with $99 \%$ confidence. (B) Structural alignment of Mdes001440 using RSP-BLAST (Altschul et al., 1997) and human serine/threonine phosphatase 2C (K.Das et al., 1996). Blue residues are structurally aligned. Red residues (near the active site) are identical and structurally aligned. Grey residues are unaligned. Grey spheres represent manganese or magnesium ions.

\subsection{Candidate $\mathrm{vH} 24$ is a member of a small effector-encoding gene family}

Searching the HF reference sequence genome revealed 17 other genes and gene fragments that have significant sequence similarity to Mdes001440 (TBLASTN $\leq 1.0 \mathrm{e}-8$ ). The sequence similarities are associated within both the PP2C motif and the secretion signal (Fig. S2). A secretion signal is lacking in three of these genes. However, gaps in the reference HF genome sequence truncate each of these genes at their $5^{\prime}$-ends, so it is likely that the signal-peptideencoding sequences of these genes are simply masked by these gaps. Two of the 17 genes identified are composed of two exons, but like Mdes001440, at least 12 others are composed of a single exon. Ten of the genes are located on chromosome A2; five of these exist in tandem on scaffold A2.12, three are clustered on scaffold A2.1 and two are located in tandem on scaffold A2.8. All 18 genes exist within the "self-homology" genic fraction of the HF genome that was previously predicted to encode effectors (Zhao et al., 2015). Thus, our map-based approach to $v H 24$ discovery appears to have uncovered a small family of putative PP2C-like effector encoding genes.

To examine the origin of this gene family, we used TBLASTN to identify putative homologs in the Drosophila melanogaster (Drosophila) genome. Interestingly, whereas two of the HF genes had no apparent homologs in the $D$. melanogaster genome, 16 others were similar in sequence to Drosophila gene CG7115 (Table 2). These similarities were present only within the PP2C domain. CG7115 lacks a secretion signal. We also used TBLASTN to search the HF genome for homologs to each of the 15 PP2C encoding Drosophila genes (Table 2). These genes include fos intronic gene (fig, CG6036), Ppm1 (CG12169), PH domain leucine rich repeat protein phosphatase (CG10493), Pyruvate dehydrogenase phosphatase (CG12151), and alphabet (alph, CG1906). The best hits to 14 of these Drosophila genes, including CG7115, do not belong to the putative PP2C-effector family (Table 2). The best hit of the Drosophila gene alph was putative HF PP2C-effector (Mde008053). However, alph is a small gene whose true 
HF ortholog may be Mdes004102 (TBLASTN = 6e-23), which is also outside of the putative HF PP2C-effector family. Taken together, these observations lead us to conclude that the putative HF PP2C-effector family arose from an expansion of an insect PP2C domain after it became aligned with a signal peptide encoding exon. Evolution of the genes in the PP2C-effector family has made Mdes001440 and two other HF genes less recognizable as insect PP2C homologs.

Table 2

TBLASTN "best hits" (BH) of PP2C encoding genes in HF and D. melanogaster (Dm) genomes.

\begin{tabular}{|c|c|c|c|c|c|c|c|}
\hline \multicolumn{4}{|c|}{ TBLASTN to Dm annotated genes } & \multicolumn{4}{|c|}{ TBLASTN to HF genome sequence } \\
\hline HF query & $\mathrm{Dm} \mathrm{BH}$ & E-value & $\mathrm{SP}^{1}$ & Dm query & $\mathrm{HF} \mathrm{BH}$ & E-value & $\mathrm{SP}^{1}$ \\
\hline Mdes001440 & CG7115 & 0.052 & No & CG7115 & Mdes008796 & $1 e-54$ & No \\
\hline Mdes007548 & CG7115 & $1 e-26$ & No & CG17598 & Mdes000375 & $1 e-176$ & No \\
\hline Mdes003813 & None & & No & CG10376 & Mdes011060 & $2 e-31$ & No \\
\hline Mdes017452 & CG7115 & $3 e-04$ & No & CG12169 & Mdes006632 & $1 e-41$ & No \\
\hline Mdes017453 & CG7115 & $5 e-07$ & No & CG17746 & Mdes006632 & $7 e-42$ & No \\
\hline Mdes008052 & CG7115 & $5 e-50$ & No & CG10493 & Mdes000801 & $9 e-14$ & No \\
\hline Mdes008053 & CG7115 & $2 e-44$ & No & CG2984 & Mdes000336 & $1 e-70$ & No \\
\hline Mdes001891 & CG7115 & $1 e-45$ & No & CG15035 & Mdes006807 & $6 e-47$ & No \\
\hline Mdes010556 & CG7115 & $3 e-46$ & No & CG9801 & Mdes007315 & $1 e-153$ & No \\
\hline Mdes017437 & CG7115 & $6 e-39$ & No & CG6036 & Mdes004102 & $2 e-22$ & No \\
\hline Mdes001889 & CG7115 & $3 e-31$ & No & CG7615 & Mdes006807 & $8 e-26$ & No \\
\hline Mdes001892 & CG7115 & $2 e-39$ & No & CG1906 & Mdes008053 & $2 e-24$ & Yes \\
\hline Mdes001893 & CG7115 & $1 e-49$ & No & CG1215 & Mdes004387 & $1 e-118$ & No \\
\hline Mdes012615 & CG7115 & $3 e-40$ & No & CG10417 & Mdes004102 & $6 e-66$ & No \\
\hline Mdes014325 & CG7115 & $2 e-22$ & No & CG12091 & Mdes006807 & $6 e-45$ & No \\
\hline Mdes010558 & CG7115 & $1 e-44$ & No & & & & \\
\hline Mdes017386 & None & & No & & & & \\
\hline Mdes015322 & CG7115 & $1 e-36$ & No & & & & \\
\hline
\end{tabular}

${ }^{1}$ Absence (No) or presence (Yes) of a secretion signal in the BH sequence (SignalP 4.1).

\section{Discussion}

Although functional assays to test Mdes001440 for effector activity have yet to be performed, we are confident that Mdes001440 is Avr gene $v H 24$ given the following observations: 1) Mdes001440 was mapped within a genomic fragment of only $230 \mathrm{~kb}$ that contains only 21 genes. 2) It was one of only four genes within that fragment encoding a secretion signal. 3) It was the only gene within that fragment displaying the type of sequence diversity expected of an effector-encoding gene. 4) It belongs to the genic fraction of the HF genome predicted to contain effector-encoding genes and the only gene in the mapped fragment that belongs to this group. 5) It is expressed in H24-avirulent first instar larvae, but is not expressed in H24-virulent first instars. 6) An insertion near the gene's promoter segregates perfectly with H24-virulence and corresponds to a loss of the gene's expression in virulent individuals.

Finally, the secretion of PP2C protein phosphatases into plant cells is congruent with effectors that redirect, or interfere, with a plant cell's ability to respond to environmental or chemical cues. PP2C proteins reverse the protein phosphorylation activities of protein kinases in stress-related signal transduction pathways (Moorhead et al., 2009, Shi, 2009). They are largely conserved among animals and appear to have been acquired by some bacteria via lateral gene transfer (Zhang and Shi, 2004). The human genome contains 18 PP2C genes with orthologs in other vertebrates (Kerk et al., 2008, Shi, 2009). Plants have a much greater abundance of PP2C encoding genes, with 80 in Arabidopsis, 90 in rice (Oryza sativa) and 130 in corn (Zea mays) (Fuchs et al., 2013, Yang et al., 2010). Few, if any, native PP2C proteins have a signal peptide, which suggests that the candidate $\mathrm{vH} 24$ protein's secretion signal is there to permit it to function as an effector in plant cells. 
Some plant PP2Cs act as co-receptors of the plant hormone abscisic acid (ABA). This suggests a potential role for the $\mathrm{vH} 24$-encoded protein as a modulator of plant development. ABA plays regulatory roles in a number of plant physiological processes. It regulates plant responses to stress and has been implicated in the mediation of responses to plant wounding and disease (Fuchs et al., 2013, Schweighofer et al., 2007, Spartz et al., 2014, Sugimoto et al., 2014). ABA binding deactivates the phosphatase acitivity of PP2C co-receptors, which ultimately derepresses ABA response genes (Hirayama and Umezawa, 2010). Therefore, the role of the $v H 24$-encoded protein may be to interfere, disrupt, or alter one or more ABA-associated pathways to the benefit of the larva. Precedence for a PP2C effector has been observed in infectious Streptococcus pyogenes bacteria that use a PP2C protein as a virulence factor in human disease (Agarwal et al., 2012).

$v H 24$ is the fourth candidate Avr gene identified in the HF. The ability of $v H 13$, the first arthropod Avr gene discovered, to elicit plant resistance was demonstrated using RNAi gene silencing (Aggarwal et al., 2014). Attempts to silence candidate Avr genes vH6, vH9 (Zhao et al., 2015) and vH24 using the same methodology were unsuccessful. Nevertheless, in perfect agreement with the gene-for-gene hypothesis, loss-of-function mutations in all four Avr genes defeat plant resistance to a different HF $R$ gene in wheat. Like $v H 13$, (Aggarwal et al., 2014), $v H 24$ was not previously observed in the transcriptome of first-instar HF salivary glands. Therefore, although examining the transcriptome of the larval salivary gland has uncovered hundreds of putative effectors (Chen et al., 2010), it has not yet revealed the full effector complement the insect uses to attack its host.

The HF PP2C-effector family to which $v H 24$ belongs is the second HF gene family associated with Avr gene mutations. The other gene family, SSGP-71, encodes plant ubiquitin-E3-ligase mimics containing F-box and leucine rich repeat (LRR) domains (Zhao et al., 2015). We suspect that these gene families exist to provide both structural diversity and functional redundancy. Structural diversity allows a low frequency of virulence-associated loss-of-function mutations to preexist in a HF population, but the loss of a single effector does not compromise the ability of an individual insect to survive. Functional redundancy allows for the loss of an effector that is recognizable within a plant genotype that expresses the cognate $R$ gene. Other effectors that are sufficiently different in structure go unrecognized even though they have the same or a similar function in the plant.

\section{Conclusions}

The ecology of host-parasite interactions puts two fundamental physiological processes in conflict: immunological mechanisms that protect the host from foreign organisms and virulence mechanisms that allow parasites to survive on their hosts. Insect-induced plant galls are fascinating manifestations of this conflict. The utility of a completely sequenced HF genome combined with the ability to perform map-based gene discovery in the insect makes the HF a potent experimental organism for plant-insect interactions and plant-gall formation. We have illustrated here how these methodologies permit the discovery of insect effectors that elicit ETI. Genetic mapping and gene expression data identified an insertion in a HF single gene ( $v H 24)$ that is perfectly associated with the ability of HFs to survive on wheat seedlings carrying the $R$ gene H24. $v H 24$ expression is also perfectly congruent with $H 24$-virulent and H24-avirulent genotypes. $v H 24$ is a member of a small family of genes that appear to encode secreted type 2 Ser/Thr protein phosphatases. This suggests the insect uses secreted PP2Cs as effectors that modulate plant defenses and development by redirecting or interfering with signal transduction 
pathways. Definitive proof that $\mathrm{vH} 24$ is the $\mathrm{H} 24$ cognate Avr gene awaits the development of a functional assay.

\section{Acknowledgements}

The authors gratefully acknowledge support for this work provided by USDA-NIFA AFRI grants 2008- 35302-18816 and 2010-03741 and a fellowship to JJS from Fulbright-Colombia. They also gratefully acknowledge technical support from Sue Cambron (USDA-ARS) and Andrew Katz. The authors also thank three anonymous reviewers who greatly improved the presentation. 


\section{References}

1. Agarwal, S., Agarwal, S., Jin, H., Pancholi, P., and Pancholi, V. (2012) Serine/threonine phosphatase (SP-STP), secreted from Streptococcus pyogenes, is a pro-apoptotic protein. The Journal of biological chemistry 287, 9147-9167.

2. Aggarwal, R., Benatti, T., Gill, N., Zhao, C., Chen, M.-S., Shemerhorn, B., ... Stuart, J.J. (2009) A BAC-based physical map of the Hessian fly genome anchored to polytene chromosomes. BMC Genomics 10, 293.

3. Aggarwal, R., Subramanyam, S., Zhao, C., Chen, M.S., Harris, M.O., and Stuart, J.J. (2014) Avirulence effector discovery in a plant galling and plant parasitic arthropod, the Hessian fly (Mayetiola destructor). PloS one 9, e100958.

4. Allen, R.L., Bittner-Eddy, P.D., Grenville-Briggs, L.J., Meitz, J.C., Rehmany, A., Rose, L.E., and Beynon, J.L. (2004) Host-parasite coevolutionary conflict between Arabidopsis and downy mildew. Science 306, 1956-1960.

5. $\quad$ Altschul, S.F., Gish, W., Miller, W., Myers, E.W., and Lipman, D.J. (1990) Basic local alignment search tool. J. Molec. Biol. 215, 403-410.

6. Altschul, S.F., Madden, T.L., Schäffer, A.A., Zhang, J., Zhang, Z., Miller, W., and Lipman, D.J.ß. (1997) Gapped BLAST and PSI-BLAST: a new generation of protein database search programs. Nucleic Acids Research 25, 3389-3402.

7. Benatti, T., Valicente, F.H., Aggarwal, R., Zhao, C., Walling, J.G., Chen, M.-S., ... Stuart, J.J. (2010) A neo-sex chromosome that drives postzygotic sex determination in the Hessian fly (Mayetiola destructor). Genetics 184, 769-777.

8. Bent, A.F. and Mackey, D. (2007) Elicitors, effectors, and $R$ genes: The new paradigm and a lifetime supply of questions. Annu. Rev. Phytopath. 45, 399-346.

9. Bos, J.I.B., Prince, D., Pitino, M., Maffei, M.E., Win, J., and Hogenhout, S.A. (2010) A functional genomics approach identifies candidtate effectors from the aphid species Myzus persicae (green peach aphid). PLoS Genet. 6, e1001216.

10. Chen, M.-S., Echegaray, E., Whitworth, R.J., Wang, H., Sloderbeck, P.E., Knutson, A., . . . Royer, T.A. (2009) Virulence analysis of Hessian fly populations from Texas, Oklahoma, and Kansas. J. Econ. Enotmol. 102, 774-780.

11. Chen, M.-S., Liu, X., Yang, Z., Zhao, H.X., Shukle, R.H., Stuart, J.J., and Hulbert, S. (2010) Unusual conservation among genes encoding small secreted salivary gland proteins from a gall midge. BMC Evolutionary Biology 10, 296.

12. Ellis, J.G., Rafiqi, M., Gan, P., Chakrabarti, A., and Dodds, P.N. (2009) Recent progress in discovery and functional analysis of effector proteins of fungal and oomycete plant pathogens. Curr. Opin. Plant Biol. 12, 1-7.

13. Fuchs, S., Grill, E., Meskiene, I., and Schweighofer, A. (2013) Type 2C protein phosphatases in plants. The FEBS journal 280, 681-693.

14. Gallun, R.L., Deay, H.O., and Cartwright, W.B. (1961) Four races of Hessian fly selected and developed from an Indiana population. Purdue Agricultrual Research Station Bulletin 732, 1-8.

15. Hao, Y., Cambron, S.E., Chen, Z., Wang, Y., Bland, D.E., Buntin, G.D., and Johnson, J.W. (2013) Characterization of new loci for Hessian fly resistance in common wheat. TAG. Theoretical and applied genetics. Theoretische und angewandte Genetik 126, 1067-1076. 
16. Harris, M.O., Freeman, T.P., Anderson, K.G., Moore, J.A., S. A. Payne, Anderson, K.M., and Rohfritsch, O. (2010) H gene-mediated resistance to Hessian fly exhibits features of penetration resistance to fungi. Phytopathology 100, 279-289.

17. Harris, M.O., Freeman, T.P., Rohfritsch, O., Anderson, K.G., Payne, S.A., and Moore, J.A. (2006) Virulent Hessian fly (Diptera: Cecidomyiidae) larvae induce a nutritive tissue during compatible interactions with wheat. Ann. Entomol. Soc. Am. 99, 305-316.

18. Harris, M.O., Friesen, T.L., Xu, S.S., Chen, M.S., Giron, D., and Stuart, J.J. (2015) Pivoting from Arabidopsis to wheat to understand how agricultural plants integrate responses to biotic stress. J Exp Bot 66, 513-531.

19. Harris, M.O., Stuart, J.J., Mohan, M., Nair, S., Lamb, R.J., and Rohfritsch, O. (2003) Grasses and gall midges: Plant defense and insect adaptation. Annu. Rev. Entomol. 48, 549-577.

20. Hirayama, T. and Umezawa, T. (2010) The PP2C-SnRK2 complex: the central regulator of an abscisic acid signaling pathway. Plant Signalling \& Behavior 5, 160163.

21. Hogenhout, S.A. and Bos, J.I. (2011) Effector proteins that modulate plant--insect interactions. Current opinion in plant biology 14, 422-428.

22. Hogenhout, S.A., Van der Hoorn, R.A.L., Terauchi, R., and Kamoun, S. (2009) Emerging concepts in effector biology of plant-associated organisms. Mol. PlantMicrobe Interact. 22, 115-122.

23. International Wheat Genome Sequencing, C. (2014) A chromosome-based draft sequence of the hexaploid bread wheat (Triticum aestivum) genome. Science 345, 1251788.

24. Jones, J.D.G. and Dangl, J.L. (2006) The plant immune system. Nature 444, 323-329.

25. K.Das, A., R.Helps, N., T.W.Cohen, P., and Barford, D. (1996) Crystal structure of the protein serine/threonine phosphatase 2C at $2.0 \AA$ A resolution. EMBO Journal 15, 6798-6809.

26. Kaloshian, I. (2004) Gene-for-gene disease resistance: bridging insect pest and pathogen defense. J. Chem. Ecol. 30, 2419-2438.

27. Kelley, L.A. and Sternberg, M.J. (2009) Protein structure prediction on the Web: a case study using the Phyre server. Nature protocols 4, 363-371.

28. Kerk, D., Templeton, G., and Moorhead, G.B. (2008) Evolutionary radiation pattern of novel protein phosphatases revealed by analysis of protein data from the completely sequenced genomes of humans, green algae, and higher plants. Plant physiology 146, 351-367.

29. Kobayashi, T., Yamamoto, K., Suetsugu, Y., Kuwazaki, S., Hattori, M., Jairin, J., ... Matsumura, M. (2014) Genetic mapping of the rice resistance-breaking gene of the brown planthopper Nilaparvata lugens. Proceedings. Biological sciences / The Royal Society 281.

30. Lee, E., Helt, G.A., Reese, J.T., Munoz-Torres, M.C., Childers, C.P., Buels, R.M., ... Lewis, S.E. (2013) Web Apollo: a web-based genomic annotation editing platform. Genome Biology 14, R93.

31. Li, C., Chen, M., Chao, S., Yu, J., and Bai, G. (2013) Identification of a novel gene, H34, in wheat using recombinant inbred lines and single nucleotide polymorphism markers. TAG. Theoretical and applied genetics. Theoretische und angewandte Genetik 126, 2065-2071. 
32. Li, G., Wang, Y., Chen, M.S., Edae, E., Poland, J., Akhunov, E., .. Y Yan, L. (2015) Precisely mapping a major gene conferring resistance to Hessian fly in bread wheat using genotyping-by-sequencing. BMC Genomics 16, 108.

33. Liu, X., Bai, J., Huang, L., Zhu, L., Liu, X., Weng, N., ... Chen, M.-S. (2007) Gene expression of different wheat genotypes during attack by virulent and avirulent Hessian fly (Mayetiola destructor) larvae. J. Chem. Ecol. 33, 2171-2194.

34. Liu, X., Khajuria, C., Li, J., Trick, H.N., Huang, L., Gill, B.S., ... Chen, M.S. (2013) Wheat Mds-1 encodes a heat-shock protein and governs susceptibility towards the Hessian fly gall midge. Nature communications 4, 2070.

35. Ma, Z.-Q., Gill, B.S., Sorrells, M.E., and Tanksley, S.D. (1993) RFLP markers linked to two Hessian fly resistance genes in wheat (Triticum aestivum, L.) from Triticum tauschii(Coss.) Schmal. TAG 85, 750-754.

36. McDonald, M.J., Ohm, H.W., Rinehart, K.D., Giovanini, M.P., and Williams, C.E. (2014) H33: A Wheat Gene Providing Hessian Fly Resistance for the Southeastern United States. Crop Science 54, 2045.

37. Milligan, S.B., Bodeau, J., Yaghoobi, J., Kaloshian, I., Zabel, P., and Williamson, V.M. (1998) The root knot nematode resistance gene $M i$ from tomato is a member of the leucine zipper, nucleotide binding, leucine-rich repeat family of plant genes. The Plant cell 10, 1307-1319.

38. Moorhead, G.B., De Wever, V., Templeton, G., and Kerk, D. (2009) Evolution of protein phosphatases in plants and animals. The Biochemical journal 417, 401-409.

39. Orcheski, B.B. and Davis, T.M. (2010) An enhanced method for sequence walking and paralog mining: TOPO(R) Vector-Ligation PCR. BMC research notes 3, 61.

40. Petersen, T.N., Brunak, S., Heijne, G.v., and Nielsen, H. (2011) SignalP 4.0: discriminating signal peptides from transmembrane regions. Nature Methods 8, 785786.

41. Raupp, W.J., Amri, A., Hatchett, J.H., Gill, B.S., Wilson, D.L., and Cox, T.S. (1993) Chromosomal location of Hessian fly-resistance genes $H 22, H 23$, and $H 24$ derived from Triticum tauschii in the D genome of wheat. J. Hered. 84, 142-145.

42. Richards, S. and Murali, S.C. (2015) Best Practices in Insect Genome Sequencing: What Works and What Doesn't. Curr Opin Insect Sci 7, 1-7.

43. Rider, S.D., Jr., Sun, W., Ratcliffe, R.H., and Stuart, J.J. (2002) Chromosome landing near avirulence gene $v H 13$ in the Hessian fly. Genome 45, 812-822.

44. Rossi, M., Goggin, F.L., Milligan, S.B., Kaloshian, I., Ullman, D.E., and Williamson, V.M. (1998) The nematode resistance gene $M i$ of tomato confers resistance against the potato aphid. Proc. Natl. Acad. Sci. USA 95, 9750-9754.

45. Schweighofer, A., Kazanaviciute, V., Scheikl, E., Teige, M., Doczi, R., Hirt, H., ... Meskiene, I. (2007) The PP2C-type phosphatase AP2C1, which negatively regulates MPK4 and MPK6, modulates innate immunity, jasmonic acid, and ethylene levels in Arabidopsis. The Plant cell 19, 2213-2224.

46. Shi, Y. (2009) Serine/threonine phosphatases: mechanism through structure. Cell $139,468-484$.

47. Shorthouse, J.D., Wool, D., and Raman, A. (2005) Gall-inducing insects - Nature's most sophisticated herbivores. Basic and Applied Ecology 6, 407-411.

48. Smith, C.M. and Clement, S.L. (2012) Molecular bases of plant resistance to arthropods. Annu. Rev. Entomol. 57, 309-328. 
49. Spartz, A.K., Ren, H., Park, M.Y., Grandt, K.N., Lee, S.H., Murphy, A.S., ... Gray, W.M. (2014) SAUR Inhibition of PP2C-D Phosphatases Activates Plasma Membrane H+ATPases to Promote Cell Expansion in Arabidopsis. The Plant cell 26, 2129-2142.

50. Staskawicz, B.J., Dahlbeck, D., and Keen, N.T. (1984) Cloned avirulence gene of Pseudomonas syringae pv. glycinea determines race-specific incompatibility on Glycine max (L.) Merr. Proc. Natl. Acad. Sci. USA 81, 6024-6028.

51. Stuart, J. (2015) Insect effectors and gene-for-gene interactions with host plants. Current Opinion in Insect Science 9, 1-6.

52. Stuart, J.J., Chen, M.S., Shukle, R., and Harris, M.O. (2012) Gall Midges (Hessian Flies) as Plant Pathogens. Annu Rev Phytopathol.

53. Sugimoto, H., Kondo, S., Tanaka, T., Imamura, C., Muramoto, N., Hattori, E., ... Ohto, C. (2014) Overexpression of a novel Arabidopsis PP2C isoform, AtPP2CF1, enhances plant biomass production by increasing inflorescence stem growth. J Exp Bot 65, 5385-5400.

54. van der Hoorn, R. and Kamoun, S. (2008) From guard to decoy: A new model for perception of plant pathogen effectors. The Plant cell 20, 2009-2017.

55. Williams, C.E., Nemacheck, J.A., Shukle, J.T., Subramanyam, S., Saltzmann, K.D., and Shukle, R.H. (2011) Induced epidermal permeability modulates resistance and susceptibility of wheat seedlings to herbivory by Hessian fly larvae. J. Exp. Botany.

56. Yang, M., Song, S., Liu, G., Chen, K., Tian, X., Zhao, Z.J., ... Yu, J. (2010) A comprehensive analysis of protein phosphatases in rice and Arabidopsis. Plant Systematics and Evolution 289, 111-126.

57. Zantoko, L. and Shukle, R.H. (1997) Genetics of virulence in the Hessian fly to resistance gene $H 13$ in wheat. J. Hered. 88, 120-123.

58. Zhang, W. and Shi, L. (2004) Evolution of the PPM-family protein phosphatases in Streptomyces: duplication of catalytic domain and lateral recruitment of additional sensory domains. Microbiology 150, 4189-4197.

59. Zhao, C., Escalante, L.N., Chen, H., Benatti, T.R., Qu, J., Chellapilla, S., ... Richards, S. (2015) A massive expansion of effector genes underlies gall-formation in the wheat pest Mayetiola destructor. Current Biology 25, 613-620.

60. Zhu, L., Liu, X., Liu, X., Jeannotte, R., Reese, J.C., Harris, M.O., ... Chen, M.-S. (2008) Hessian fly (Mayetiola destructor) attack causes a dramatic shift in carbon and nitrogen metabolism in wheat. Mol. Plant-Microbe Interact. 21, 70-78. 